\title{
1 Molecular evidence of outcrossing rate variability in Brassica napus
}

2

3 P. Soengas ${ }^{1}$, G. Padilla, M. Francisco, P. Velasco, and M.E. Cartea

$4{ }^{1}$ Department of Plant Genetics, Misión Biológica de Galicia (CSIC), Apartado 28, E-

536080 Pontevedra, Spain.

6

7 Research supported by the Committee for Science and Technology of Spain (AGL2006-

8 04055) and Excma. Diputación Provincial de Pontevedra, Spain.

9

10 Received:

11

12 To whom reprints should be addressed. e-mail address:

13 psoengas@mbg.cesga.es

14 TLF: 0034986854800

15 


\section{ABSTRACT}

18 A leafy crop of Brassica napus L. called nabicol has been grown by farmers in

19 northwestern Spain for many years, being an important horticultural product during the

20 winter season. A collection of landraces of a Brassica napus leafy crop called nabicol is

21 kept at 'Misión Biológica de Galicia' (CSIC-Spain) which can be used to search for

22 desirable characteristics or to produce new commercial varieties to release in the market

23 . The assessment of the mating system of nabicol landraces is particularly important to

24 carry on adequate breeding and genetic conservation programs. The objective of this

25 work is to estimate the outcrossing rate in nabicol under controlled pollinator conditions

26 using SSRs. Pairs of flowering plants taken from nabicol landrace MBG-BRS0039 were

27 placed in separated isolation cages and bumble bees (Bombus $s p$ ) were released for

28 facilitating the crosses between plants. A seed sample from each plant in the cross was

29 analyzed by SSRs that were polymorphic in the parental population. We found that the

30 crop is partially allogamous and that there is genetic variation for the outcrossing rate

31 among individuals. Several consequences for the maintenance and genetic improvement

32 of the crop are discussed. The existence of genetic variability for this trait is a valuable

33 tool that will allow us to study the genetic mechanisms underlying the mating system of

34 this crop.

36 Keywords: Brassica napus, nabicol landraces, mating system, outcrossing rate, 37 microsatellites, SSRs 
INTRODUCTION

42 Brassica napus includes economically important crops, such as oilseed rape (B. napus

43 L. group oleifera DC.), rutabaga (B. napus group napobrassica L. Reichenb.) and leaf

44 rape (vegetable and fodder crops) (B. napus L. group pabularia (DC.) Reichenb.).

45 Traditionally B. napus has been considered an autogamus species, although some

46 authors have found that in field conditions, it can show a variable level of outcrossing.

47 Estimates in oilseed rape were found to vary between 20-40\% (Olsson 1960; Rakow

48 and Woods 1987; Becker et al. 1992; Damgaard and Loeschcke 1994). In rutabaga, the

49 percentage of outcrossing varies between $20-45 \%$ (Gowers 1981). As far as we know,

50 no studies have been done in leafy B. napus crops. Breeding of partially allogamous

51 crops is very difficult. Therefore, in B. napus selfing is widely used in early generations

52 and standard methods like pedigree selection or single seed descendent are applied. The

53 high demand for uniformity, distinctness and stability strongly favor pure lines or $F_{1}$

54 hybrids as the desired type of cultivar (Becker et al. 1999).

A leafy crop of B .napus called nabicol has been grown by farmers in

56 northwestern Spain for many years, being an important horticultural product during the

57 winter season (Cartea et al. 2005). The leaves are used for human consumption (in

58 soups and in stews) and fodder. Local nabicol landraces from northwestern Spain were

59 collected since the 1980 s until the present and they are currently kept as an active

60 collection at the 'Misión Biológica de Galicia' (CSIC, Spain). They are stored at $4{ }^{\circ} \mathrm{C}$

61 and less than 50\% humidity. The molecular, morphological and agronomical

62 characteristics of this collection have been previously studied (Cartea et al. 2005;

63 Rodriguez et al. 2005; Soengas et al. 2006). Some landraces were considered promising

64 for fresh production because they showed better or similar yield than commercial 
65 controls (Rodriguez et al. 2005) and they could be included in breeding programs to

66 obtain improved varieties. Nabicol landraces represent a valuable resource to obtain

67 resistant varieties to pest and diseases and to produce new commercial varieties. In order to be able to manage germplasm effectively, some basic information is

69 required. This includes information on the nature of mating system (Hamilton et al.

70 2003), meaning the extent of self-pollination and the degree of outcrossing. To design

71 breeding programs it is necessary to determine the mating system of the crop under

72 study. Currently, accurate estimates of outcrossing rates can be achieved using neutral

73 molecular markers, such as microsatellites (SSRs). The advantage of using this kind of

74 markers is that they are not subjected to the action of natural or human selection, like

75 morphological traits (for example flower color, which can be biased because of

76 pollinator preferences). Estimates of outcrossing rates can also be obtained using several

77 SSRs at the same time. Compared with single-locus methods, multi-locus methods have

78 lower statistical variance and higher robustness against the violation of model

79 assumptions (Ritland and Jain 1981). The multi-locus method proposed by Ritland and

80 Jain is an iterative procedure which uses the Newton-Raphson method to compute

81 outcrossing rates. The program is based on the mixed-mating model of Brown and

82 Allard (1970) that has several assumptions: 1) mating events are due to either selfing or

83 random outcrossing, 2) maternal individuals sample pollen from an homogeneous

84 pollen pool, 3) outcrossing rates are uniform over all maternal individuals within a

85 population and 4) there is no selection between fertilization and time of assay.

86 The objective of this work was to estimate the outcrossing rate in a leafy crop of

87 B. napus under controlled pollination conditions using the SSR multi-locus method of

88 Ritland and Jain (1981). 
MATERIAL AND METHODS

91

92 To determine the outcrossing rate of nabicol, the 35 nabicol landraces currently kept at

93 'Misión Biológica de Galicia' (CSIC, Spain) were initially studied. Thirty plants of each

94 landrace were screened using 40 SSRs (www.ukcrop.net). Most landraces were

95 monomorphic for the SSRs studied, so they were discarded. From the remaining

96 landraces, MBG-BRS0039 was chosen to carry on the experiments because it was the

97 only one whose individuals showed a synchronic flowering. MBG-BRS0039 had shown

98 good early vigor, good leaf and seed yield and intermediate growing cycle compared to

99 other landraces from the collection in a previous characterization carried out by

100 Rodriguez et al. (2005). Eight pairs of synchronously flowering plants from MBG-

101 BRS0039 were placed in separate isolation cages, where bumble bees (Bombus sp) were

102 released to facilitate pollination of both parents.

A seed sample from each plant was harvested and all the seed harvested in each

104 plant was considered as a 'family'. Thus, 16 families were obtained to perform the

105 statistical analysis. Approximately 100 seeds of each family were sown in seedbeds and

106 the 4 or 5 youngest leaf of each individual plant were collected 40 days after sowing.

107 Individual plants from each family were genotyped by those SSRs that had been

108 polymorphic in the parental population.

109 DNA of each individual was extracted following the method of Liu and

110 Whittier (1994) with minor modifications. Amplifications were performed by using a

111 PTC- $100^{\mathrm{TM}}$ Thermal Cycler (MJ Research, Watertown, MA). The amplification

112 consisted of a denaturing step at $95^{\circ} \mathrm{C}$ for 5 min followed by 35 cycles at $95{ }^{\circ} \mathrm{C}$ for 30

$113 \mathrm{sec}, 56^{\circ} \mathrm{C}$ for $30 \mathrm{sec}$, and $72{ }^{\circ} \mathrm{C}$ for $30 \mathrm{sec}$. The program ended with an extra elongation

114 period of $10 \mathrm{~min}$ followed by a continuous cycle at $4{ }^{\circ} \mathrm{C}$. PCR reactions were carried out 
115 in a volume of $25 \mu \mathrm{l}$ containing $50 \mathrm{ng}$ of each primer, 0.6 unit of Taq polymerase

116 (ECOGEN), $200 \mu \mathrm{M}$ each dNTP, $1 \mathrm{X}$ reaction buffer, $2.0 \mathrm{mM} \mathrm{MgCl} 2,50$ ng DNA

117 template, in distilled and autoclaved water. After amplification, SSR products were

118 separated by electrophoresis on $6 \%$ non-denaturing acrylamide gels run in $1 \times \mathrm{TBE}$

119 buffer. Gels were stained with Ethidium bromide, run for approximately $2 \mathrm{~h}$ at $250 \mathrm{~V}$,

120 and then were visualized under UV light.

121 Estimates of outcrossing rate were computed using the iterative procedure of

122 Ritland and Jain (1981). This method assumes a 'mixed mating model' of a population in

123 which, the progeny of any plant is the result of self-fertilization and outcrossing.

124 Maximum likelihood estimates of outcrossing rates were obtained at population level

125 with the Newton-Raphson method (Ritland and Jain 1981). Standard errors were

126 calculated by bootstraps (1000) using the families as the resampling unit. Using this

127 method, single-locus outcrossing rates $\left(t_{s}\right)$ and multi-locus outcrossing rates $\left(t_{m}\right)$ were

128 estimated. The difference between $t_{m}$ and the $t_{s}$ averaged across loci, and the correlation

129 of outcrossing rate estimates among loci were also computed. Estimates of outcrossing

130 rates at a family level were computed with a method of moments, as described by Ritland

131 and Jain (1981).

132 Two different statistical approaches were used to analyze the data. The method of

133 Ritland and Jain (1981) can be implemented for both diploid and tetraploid species.

134 When a microsatellite amplified only one locus or several loci that were clearly

135 distinguishable, then they were scored and analyzed using the method for diploid species.

136 The program MLTR version 3.2 was used to analyze the data

137 (http://genetics.forestry.ubc.ca/ritland/programs.html). If a SSR amplified two loci,

138 whose alleles could not be assigned to any of both loci the method of Ritland and Jain

139 (1981) for tetraploid species was used to score and analyze the data. The program 
140 MLTET (http://genetics.forestry.ubc.ca/ritland/programs.html) was used in this case 141 (Ritland 1990; Ritland 2002).

142 


\section{RESULTS AND DISCUSSION}

145 Results from amplification in the parental population showed that three SSRs, Na12-A02,

146 Na14-D07 and Na10-A08 were polymorphic (Table 1). SSRs primer sequences and

147 annealing temperatures are reported by Lowe et al. (2004). SSR Na10-A08 amplified two

148 different, polymorphic and independent loci: Na10-A08a, which was localized in a

149 linkage group of B. napus (N15), and equivalent to the Brassica rapa linkage group R15

150 and Na10-A08b (N9) which was mapped in the equivalent Brassica oleracea linkage

151 group 09 (Piquemal et al. 2005). The SSR Na12-A02 amplified two different loci but this

152 primer showed polymorphic banding for only one of them. SSR Na14-D07 amplified two

153 different loci with their corresponding alleles running at the same height.

154 SSR Na14-D07, SSR Na10-A08a and Na10-A08b amplified two alleles each, while

155 Na12-A02 amplified four alleles in the parental population. Allele frequencies for each

156 SSR marker are presented in Table 1. Frequency of heterozygote genotypes were among

$15716 \%$ for SSR Na12-A02 and 39\% for SSR Na14D07.

158 The outcrossing rate of MBG-BRS0039 was computed using two different

159 approaches. The MLTET program was using to compute the outcrossing rate with SSR

160 Na14-D07while for the remaining loci MLTR program was employed (Ritland 1990;

161 Ritland 2002). The idea of using two different approaches comes from the difficulty of

162 scoring some SSRs, when more than one locus is being amplified with the same primers.

163 This problem has been described before and is a result of the complex structure of the

164 Brassica genome. B. napus (AACC) is an amphidiploid between B. rapa (AA) and B.

165 oleracea (CC). Sometimes, homologous loci can be found inside the B. oleracea and $B$.

166 rapa genomes and the assignment of alleles to loci is difficult and, in some cases, 
167 impossible. Furthermore, homologous B. napus SSR loci can display identical alleles that 168 cannot be resolved (Hasan et al. 2006).

169 The outcrossing rate computed using the MLTET program, was $0.30 \pm 0.13$

170 (Table 2). The estimation of the outcrossing rates at the loci Na12-A02, Na10-A08a and

171 Na10-A08b were computed with the program MLTR. The program produces an

172 estimator of the single-locus outcrossing rate $\left(t_{s}\right)$, and the multi-locus outcrossing rate

$173\left(t_{m}\right)$. The values of $t_{s}$ were very similar for the three loci examined, but were more

174 precise when the estimate was computed with the loci Na10-A08a than with the other

175 two (Table 2). The combined value of $t_{m}$ for the loci Na12-A02, Na10-A08a and Na10-

176 A08b was equal to $0.33 \pm 0.10$, which is not significantly different from that detected at

177 the locus SSR Na14-D07. Similar estimates of $t_{s}$ were obtained with the methods

178 implemented for diploid and tetraploid species, indicating that both were adequate to 179 analyze the genome of $B$. napus.

180 The $t_{m}$ value found in the nabicol landrace MBG-BRS0039 is in agreement with

181 those detected in other B. napus crops. Olsson (1960) found that on average, there is

182 about one-third cross-fertilization and two-thirds self-fertilization in rapeseed using

183 morphological traits. Becker et al. (1992) found similar values of outcrossing in

184 rapeseed (an average outcrossing rate of 34\%) using isozyme analysis. In rutabaga, the

185 level of outcrossing determined by Gowers (1981) using morphological traits was

186 similar to those found in oilseed rape.

187 This work demonstrates that nabicol crop has a similar level of outcrossing that

188 other B. napus crops. The crop can be therefore considered as partially allogamous. The 189 accessions of the nabicol collection of the MBG are regenerated from time to time in 190 order to get enough seed to allow us to carry out different trials and send seed to other 191 researchers or growers when requested. In order to maintain the genetic integrity of the 
192 accessions as much as possible, they must be multiplied by using the same methods

193 used for allogamous Brassica crops. In this case, isolation conditions are necessary to

194 avoid gene flow and the minimum number of plants multiplied for each accession must

195 be higher than in the case of self-pollinating crops to preserve the variability present.

196 Estimates of $t_{s}$ and $t_{m}$ were also computed at a family level. The number of

197 individuals analyzed by family varied depending on the loci studied. This variation in

198 the sample size was due to missing data for some of the loci when PCR amplifications

199 for some individuals did not performed well and to the presence of possible null alleles.

200 Even using large sample sizes, some estimates of $t_{s}$ in the four loci examined were

201 above 1.00. For example, in families 14 and 15 for the locus Na14-D07 (Table 2)

202 estimates were over 1.00. Averaged $t_{\mathrm{s}}$ across loci or $t_{\mathrm{m}}$ estimates were below or close to

2031.00 , proving that multi-locus estimates are more accurate than single locus estimates.

204 The $t_{m}$ computed at a family level gave estimates that ranged from values close to 0.00

205 (families number 2 and 13) to values close to 1.00 (families 8 and 15). Most families

206 showed outcrossing rates between 0.20 and 0.40 (Table 2). The biparental inbreeding

207 was estimated as the difference between $t_{m}$ and $t_{s}$ averaged across loci $(0.04 \pm 0.02)$.

208 When the biparental inbreeding is present, it can cause a bias in the estimation of selfing,

209 but, in our case, this bias was almost negligible.

210 The correlation among outcrossing rates estimates obtained with different loci

211 and computed within progeny arrays $\left(\mathrm{r}_{\mathrm{t}}\right)$ was $0.24 \pm 0.18$. This correlation is low,

212 indicating that outcrossing rate is dependent on the locus employed, proving again that

213 multi-locus approaches give more accurate results than single-locus approaches.

214 Variability for outcrossing rate could be found among individuals of MBG-

215 BRS0039. With the experimental design employed, we could not separate the genetic

216 and the environmental components of the phenotypic variability. We only could make 
217 some assumptions on the causes of this variability in the present work. Phenotypic

218 variation is due in part to environmental factors, such as the position of the flowers in

219 the plant (Becker et al. 1992) or the preference and number of visits of pollinators (Suso

220 et al. 2005). However, in our experiment, part of the environmental variation was

221 reduced by placing pairs of plants in isolation cages. Different isolation cages were used

222 for each pair of plants, and within these cages, beehives with approximately the same

223 number of bumble bees were placed. These steps were taken to avoid variation based on

224 pollinator preference for a particular type of plant. It is likely that part of the variation

225 for outcrossing rate is under genetic control. Daamgard and Loeschcke (1994) estimated

226 that the narrow-sense heritability of the rate of selfing in rapeseed was 0.41 . Previously

227 reported genetic variability of outcrossing rate in rapeseed populations of B. napus

228 could have been based on differences in morphological parameters such as the spatial

229 relationship between stigmas and anthers (Syafaruddin et al. 2006) and the presence of

230 male-sterile plants (Song et al. 2006) or auto-incompatible plants (Mohring et al. 2005).

231 Differences in outcrossing rates among progeny arrays can be also overestimated since a

232 limited number of biparental crosses were chosen to estimate the outcrossing rate.

233 Finding variability for this trait is the first step to establishing an in-depth study on the

234 mating system of the crop.

235 Our results showed that nabicol is a partially allogamous crop, and that there is

236 variability for outcrossing rates among individuals. Part of this variation could be under

237 genetic control although an exhaustive study on this subject is necessary. The variability

238 found for this trait, as it was shown in this work, will allow for the study of the genetic

239 mechanisms controlling the mating system of this crop; will allow knowing how

240 heterosis and inbreeding depression are affected by the outcrossing rate, and the effect

241 on production. If the causes of the outcrossing rate and the consequences in heterosis for 
242 yield components could be determined, breeding of the crop could also exploit heterosis

243 for traits related to the production to obtain improved varieties or $F_{1}$ hybrids. To study

244 in depth causes of the outcrossing rate it is necessary to find variability for this trait. The

245 selection of self-sterile or auto-incompatible plants will become a genetic resource that

246 could be used for breeding purposes.

247 
248 Table 1. Allelic frequencies; homozygotes and heterozygotes frequencies of the three

249 SSRs analyzed in the parental population MBG-BRS0039.

\begin{tabular}{|c|c|c|c|c|}
\hline & & & Homozygotes & Heterozygotes \\
\hline Locus & Alleles & Allelic frequency & frequency & frequency \\
\hline \multirow[t]{2}{*}{ Na14-D07 } & 1 & 0.750 & 0.612 & 0.388 \\
\hline & 2 & 0.250 & & \\
\hline \multirow[t]{2}{*}{ Na10-A08a } & 1 & 0.796 & 0.794 & 0.206 \\
\hline & 2 & 0.203 & & \\
\hline \multirow[t]{2}{*}{$\mathrm{Na} 10-\mathrm{A} 08 \mathrm{~b}$} & 1 & 0.783 & 0.776 & 0.224 \\
\hline & 2 & 0.217 & & \\
\hline \multirow[t]{4}{*}{$\mathrm{Na} 12-\mathrm{A} 02$} & 1 & 0.754 & 0.844 & 0.156 \\
\hline & 2 & 0.167 & & \\
\hline & 3 & 0.010 & & \\
\hline & 4 & 0.069 & & \\
\hline
\end{tabular}


250 Table 2. Estimates of single-locus outcrossing rate $\left(t_{s}\right)$ and multi-locus outcrossing rate $\left(t_{m}\right)$. Number of individuals of each family employed in 251 the statistical analysis using four different loci.

\begin{tabular}{|c|c|c|c|c|c|c|c|c|c|c|c|}
\hline & Na14-D07 & & Na10-A08a & & Na10-A08b & & Na12-A02 & & & & \\
\hline Family & $t_{s}{ }^{x}$ & No. ${ }^{z}$ & $t_{s}{ }^{x}$ & No. ${ }^{z}$ & $t_{s}{ }^{x}$ & No. ${ }^{z}$ & $\mathrm{t}_{\mathrm{s}}^{\mathrm{x}}$ & No. ${ }^{z}$ & Average $t_{s}{ }^{x}$ & $\mathrm{t}_{\mathrm{m}}^{\mathrm{y}}$ & No. ${ }^{\mathrm{z}}$ \\
\hline 1 & $0.32 \pm 0.17$ & 81 & $0.25 \pm 0.27$ & 81 & $1.82 \pm 0.09$ & 79 & $0.00 \pm 0.00$ & 95 & $0.57 \pm 0.09$ & $0.82 \pm 0.09$ & 95 \\
\hline 2 & $0.20 \pm 0.06$ & 94 & $0.26 \pm 0.11$ & 94 & $0.05 \pm 0.05$ & 95 & $0.00 \pm 0.00$ & 95 & $0.12 \pm 0.05$ & $0.14 \pm 0.06$ & 95 \\
\hline 3 & $0.50 \pm 0.09$ & 76 & $1.28 \pm 0.23$ & 84 & $0.80 \pm 0.05$ & 84 & $0.00 \pm 0.00$ & 84 & $0.76 \pm 0.14$ & $0.66 \pm 0.16$ & 84 \\
\hline 4 & $0.02 \pm 0.00$ & 94 & $0.57 \pm 0.16$ & 94 & $0.17 \pm 0.30$ & 92 & $0.00 \pm 0.00$ & 95 & $0.17 \pm 0.10$ & $0.33 \pm 0.17$ & 95 \\
\hline 5 & $0.67 \pm 0.10$ & 84 & $0.76 \pm 0.19$ & 90 & $0.00 \pm 0.01$ & 95 & $1.63 \pm 0.29$ & 87 & $0.66 \pm 0.09$ & $0.78 \pm 0.11$ & 95 \\
\hline 6 & $0.34 \pm 0.07$ & 94 & $0.45 \pm 0.27$ & 81 & & & $0.69 \pm 0.22$ & 78 & $0.79 \pm 0.20$ & $0.38 \pm 0.21$ & 82 \\
\hline 7 & $1.19 \pm 0.67$ & 73 & $0.18 \pm 0.29$ & 73 & $0.19 \pm 0.35$ & 64 & $0.56 \pm 0.04$ & 72 & $0.54 \pm 0.04$ & $0.60 \pm 0.06$ & 73 \\
\hline 8 & $0.24 \pm 0.08$ & 70 & $0.58 \pm 0.19$ & 68 & $0.00 \pm 0.01$ & 70 & $1.05 \pm 0.06$ & 70 & $0.93 \pm 0.05$ & $1.03 \pm 0.06$ & 70 \\
\hline 9 & $0.43 \pm 0.08$ & 85 & $0.46 \pm 0.28$ & 78 & $0.00 \pm 0.00$ & 84 & $0.00 \pm 0.00$ & 85 & $0.15 \pm 0.09$ & $0.29 \pm 0.17$ & 85 \\
\hline 10 & $0.00 \pm 0.14$ & 72 & $0.62 \pm 0.19$ & 71 & & & $0.00 \pm 0.00$ & 71 & $0.35 \pm 0.11$ & $0.31 \pm 0.12$ & 71 \\
\hline 11 & $0.00 \pm 0.00$ & 83 & $0.24 \pm 0.12$ & 81 & $0.23 \pm 0.30$ & 76 & $0.49 \pm 0.19$ & 76 & $0.31 \pm 0.12$ & $0.31 \pm 0.18$ & 83 \\
\hline
\end{tabular}




$\begin{array}{llllllllllll}12 & 0.15 \pm 0.06 & 81 & 0.22 \pm 0.06 & 76 & & & 1.92 \pm 1.37 & 72 & 0.42 \pm 0.06 & 0.48 \pm 0.06 & 76 \\ 13 & 0.53 \pm 0.11 & 52 & 0.30 \pm 0.13 & 81 & 0.16 \pm 0.33 & 78 & 0.00 \pm 0.01 & 81 & 0.06 \pm 0.12 & 0.04 \pm 0.20 & 81 \\ 14 & 1.99 \pm 0.05 & 94 & 0.51 \pm 0.16 & 86 & 0.10 \pm 0.07 & 88 & 0.00 \pm 0.00 & 88 & 0.29 \pm 0.08 & 0.33 \pm 0.09 & 88 \\ 15 & 1.99 \pm 0.01 & 88 & 0.00 \pm 0.01 & 54 & 0.56 \pm 0.35 & 50 & 1.15 \pm 0.15 & 54 & 2.57 \pm 1.76 & 0.91 \pm 0.20 & 56 \\ 16 & 0.00 \pm 0.00 & 94 & 0.10 \pm 0.10 & 48 & 0.00 \pm 0.01 & 48 & 0.26 \pm 0.13 & 95 & 0.23 \pm 0.11 & 0.24 \pm 0.11 & 95 \\ \text { Total } & 0.30 \pm 0.13 & 800 & 0.31 \pm 0.07 & 1240 & 0.30 \pm 0.14 & 1003 & 0.28 \pm 0.16 & 1298 & 0.29 \pm 0.10 & 0.33 \pm 0.10 & 1324\end{array}$

$252{ }^{\mathrm{x}}$ : single locus outcrossing rate.

253 y: multilocus outcrossing rate.

$254 \quad{ }^{\mathrm{z}}$ : number of individuals. 


\section{ACKNOWLEDGMENTS}

256

257 Research supported by the Plan Nacional I+D+I (AGL2006-04055 and AGL2009-

258 09922) and the 'Excma. Diputación Provincial de Pontevedra', Spain. G. Padilla

259 acknowledges a fellowship from the Cabildo Insular de la Palma, Spain. M. Francisco

260 acknowledges a fellowship from the I3P program, Spain. Authors want to thank the

261 invaluable help of Elisa Santiago and Rosaura Abilleira.

262 


\section{REFERENCES}

Becker HC, Damgaard C, Karlsson B (1992) Environmental variation for outcrossing rate in rapeseed (Brassica napus). Theor. Appl. Genet. 84:303-306.

Becker HC, Löptien H, Röbbelen G (1999). Breeding: an overview. In: Gómez-Campo C (ed.). Biology of Brassica coenospecies. Elsevier Science B.B. Amsterdam, The Netherlands, pp 413-460.

Brown AHD, Allard RW (1970) Estimation of the mating system in open-pollinated maize populations using isozyme polymorphisms. Genetics 66:133-145.

Damgaard C, Loeschcke V (1994) Genetic variation for selfing rate and the dependence of selfing rate on mating history in Brassica napus (rape seed). Heredity 72: $570-573$.

Cartea ME, Soengas P, Picoaga A, Ordás A (2005) Relationships among Brassica napus (L.) germplasm from Spain and Great Britain as determined by RAPD markers. Genet. Resour. Crop Evol. 52:655-662.

Gowers S (1981) Self-pollination in Swedes (Brassica napus ssp rapifera) and its implications for cultivar production. Euphytica 30:813-817.

Hamilton RS, Engels J, van Hintum T (2003) Considerations for improved conservation and utilization concepts and strategies. In: Engels JMM and Visser L (ed.). A guide to effective management of germplasm collections. IPGRI Handbooks for Genebanks No. 6. IPGRI, Rome, Italy. pp 43-56.

Hasan M, Seyis F, Badani AG, Pons-Kühnemann J, Friedt W, Lühs W, Snowdon RJ (2006) Analysis of genetic diversity in the Brassica napus L. gene pool using SSR markers. Genet. Resour. Crop Evol. 52:655-662. 
287 Liu YG, Whittier RF (1994) Rapid preparation of megabase plant DNA from nuclei in agarose plugs and microbeads. Nucleic Acid Res. 22: 2168-2169.

Lowe AJ, Moule C, Trick M, Edwards KJ (2004) Efficient large-scale development of microsatellites for marker and mapping applications in Brassica crop species. Theor. Appl. Genet. 108: 1103-1112.

292 Mohring S, Horstmann V, Esch E (2005) Development of a molecular CAPS marker for the self-incompatibility locus in Brassica napus and identification of different $\mathrm{S}$ alleles. Plant Breed.124:105-110

Olsson G (1960) Self-incompatibility and outcrossing in rape and white mustard. Hereditas 46: 241-252.

297 Piquemal J, Cinquin E, Couton F, Rondeau C, Seignoret E, Doucet I, Perret D, Villeger MJ, Vincourt P, Blanchard P (2005) Construction of an oilseed rape (Brassica napus L.) genetic map with SSR markers. Theor. Appl. Genet. 111: 1514-1523

Rakow G, Woods D (1987) Outcrossing in rape and mustard in Saskatchewan prairie conditions. Can. J. Plant Sci. 67:147-151.

302 Ritland K, Jain J (1981) A model for the estimation of outcrossing rate and gene frequencies using $n$ independent loci. Heredity 47:35-52.

304 Ritland K (1990) A series of FORTRAN computer programs for estimating plant mating systems. J. Heredity. 81:235-237.

Ritland K (2002) Extension of models for the estimation of mating systems using $n$ independent loci. Heredity 88:221-228.

308 Rodriguez VM, Cartea ME, Padilla G, Velasco P, Ordás A (2005) The nabicol: a horticultural crop in northwestern Spain. Euphytica 142: 237-246. 
310 Song LQ, Fu TD, Tu JX, Ma CZ, Yang GS (2006) Molecular validation of multiple

311 allele inheritance for dominant genic male sterility gene in Brassica napus L.

312 Theor. Appl. Genet. 113:52-62.

313 Soengas P, Velasco P, Padilla G, Ordás A, Cartea ME (2006) Genetic relationships

314 among Brassica napus crops based on SSR markers. HortScience 41:1195-1199.

315 Suso MJ, Harder JL, Moreno MT, Maalouf F (2005) New strategies for increasing

316 heterozygosity in crops: Vicia faba mating system as a study case. Euphytica $317 \quad 143: 51-65$.

318 Syafaruddin, Horisaki A, Niikura S, Yoshioka Y, Ohsawa R (2006) Effect of floral

319 morphology on pollination in Brassica rapa L. Euphytica 149:267-272. 